\title{
Not just a job, but a career
}

\author{
Adrienne Ferguson Duran, BAS, LVT, LATG, CMAR, Animal Resources \\ Manager, Section of Compliance and Rodent Clinical Care, MD Anderson \\ Cancer Center, Houston, TX
}

Mrs. Duran discusses her many responsibilities as an animal resources manager and reveals how she keeps her staff motivated.

\begin{abstract}
What originally inspired your interest in laboratory animal science?

When I was training to become a veterinary technician, we had one semester of coursework in laboratory animal medicine. At that time, I was happily working in a small animal private practice, however, and I never thought I would switch to the laboratory animal science field. I first became truly interested in the field during a school job fair, because I was attracted by the regular hours and the benefits, but I still thought I would eventually return to private practice. Now, I feel that I am making a positive difference in the lives of laboratory animals, improving the quality of research and indirectly helping people, too. My job in laboratory animal science is not just a job anymore; it is my career, and I can't see myself doing anything else.
\end{abstract}

Tell us more about what you do in your current position.

MD Anderson Cancer Center has a very large and diverse animal program with more than 500 animal protocols, affording me the opportunity to do a variety of things in my role as animal resources manager. A large part of my job is to train investigators as well as technical staff on methodologies; regulations; and pre-, peri- and postoperative surgical care for rodents in order to ensure compliance with protocols and with applicable animal welfare regulations. I work with our department's education specialist in developing training programs for our staff. I also teach American Association for Laboratory Animal Science (AALAS) certification preparation classes at all levels. I attend IACUC meetings and help the IACUC with its compliance functions by participating in semiannual inspections, providing post-approval monitoring of technical and surgical procedures and documenting the technical proficiencies of staff members. I manage the animal resources technologists who provide the daily healthcare and treatments to all of our rodents, coordinate animal imports and exports and maintain the sentinel program. Part of managing rodent healthcare includes researching the most current analgesia and enrichment strategies so that I can make recommendations to the veterinarians and investigators. I also keep our treatment algorithm up to date with the latest therapies. On several occasions, I've been asked by investigators to assist with specific projects; this has provided opportunities for me to directly assist in research.

\section{With your wide range of experience with different animal species, what are some of your favorite animal species to work with? \\ Presently, I only work with rodents, but in my previous positions I have worked with many species, from zebrafish to primates. I loved working with pigs-they are so intelligent and always enjoy different types of treats and enrichment. I also enjoyed watching and laughing at the playful antics of the cynomolgus monkeys. I think mice and rats are cute, too.}

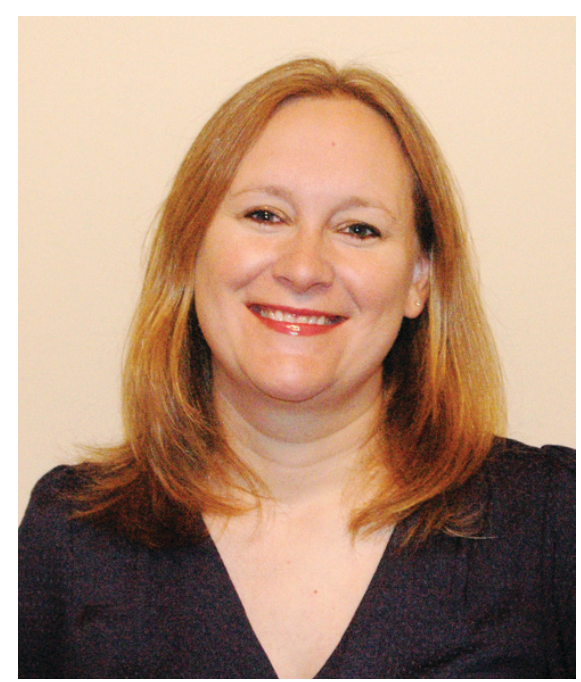

What are some of the challenges you face in your role as manager of the rodent veterinary technicians at your facility?

First, I have a staff of very intelligent people, so one of the challenges I face is keeping them intellectually stimulated and motivated. I try to consistently teach them new skills and, whenever possible, to give them opportunities to do things outside of their usual tasks. Second, we are very busy at times, and it can be a challenge just to complete the daily tasks as well as fulfill all the requests for methodological training and for technical assistance that we receive.

\section{Can you share with us some of your future goals for your career?}

In the future, I would like to have the opportunity to attend the Institute for Laboratory Animal Management course provided by AALAS. I currently serve on the Board for the Texas Branch of AALAS, but I would like to get more involved at the national level. Finally, I hope to one day become an assistant director or an associate director of an animal facility. 